\title{
Unrelated Parallel Machines Scheduling with the Effect of Aging and Learning under Multi Maintenance Activities
}

\author{
E. Ebrahimi ${ }^{1}$, J.Rezaeian ${ }^{2, *}$ \\ ${ }^{1}$ College Engineering Mazandaran University of Science and Technology. Iran \\ ${ }^{2}$ Department of Industrial Engineering, Mazandaran University of Science and Technology, Iran
}

Copyright $@ 2015$ Horizon Research Publishing All rights reserved.

\begin{abstract}
Maintenance is important to manufacturing process with a view to sustaining production efficiency. In this study scheduling of unrelated parallel machines with effects of learning and aging and multi maintenance activities is considered. The objective is to find the optimal maintenance periods, optimal maintenance frequencies and optimal job sequences such that the makespan of all jobs are minimized. Polynomial algorithm is provided and two hueristic algorithms are presented. Computational experiments are conducted to evaluate their performances.
\end{abstract}

Keywords Unrelated Parallel Machines, Learning Effect, Aging Effect, Maintenance Activities, Makespan

\section{Introduction}

Traditionally, the processing time of jobs is assumed to be fixed and constant values. In practice the actual processing time of a job maybe subject to change due to effects of learning and aging. Gupta and Gupta [14] were the first introduce deteriorating jobs in the scheduling problems. Mosheiov [13] considered the single machine problem under linear deterioration for minimizing the total completion time where the processing time of the jobs are the same. It shows that the optimal schedule is V-shaped. Lee [16] was the pioneer to introduce the learning and deterioration effects simultaneously in scheduling problems .Cheng, et al [9] investigated a new scheduling model with learning effect deterioration jobs and setup times. They proposed an optimal solution for single-machine problem in polynomial-time. Wang and Wang [8] provided resource allocation scheduling with considering learning effects, where the job's processing time is a function of the job's position in a sequence and its resource allocation. They suggested a polynomial-time algorithm to solve the optimal job sequence and resource allocation. Zhang and Yan [7] studied a learning effect model and find optimal solution for single machine and flowshop problem .Lee and Lai [6] explored a model with effects of learning and deterioration which the processing time of a job is a general function of the normal processing times of the jobs already processed and its scheduled positions.

In a real manufacturing process, machine may not be available due to preventive maintenance, periodic repairs, tools changes or breakdown so a machine may require multi-maintenance activities to improve its production efficiency or product quality. The problem of joint scheduling and maintenance has become a popular topic and has received the attention of many researchers. The reader can refer to Schmidt [17] and Ma et-al [18]. Kuo and Yang [3] considered single-machine scheduling problems with aging effects and multi-maintenance activities in acyclic process. They studied the problem with job independent and position dependent aging effect to minimize the makespan and propose an algorithm to solve the problem in polynomial time. Zhao and Tang [4] extended the investigation of Kuo and Yang [3] in addition to job dependent aging effect. They solved the problem in polynomial-time. Yang and Yang [5] considered single machine scheduling problem with the deterioration effect under a deterioration maintenance activity consideration. They suggested an algorithm to solve in polynomial time. Toksari and Guner [10] considered a parallel machine earliness and tardiness scheduling problem with the effect of learning and deterioration under different penalties. Chen [20]studied a single machine scheduling problem to minimize the number of tardy jobs under periodic maintenance activities and non-resumable jobs. He investigated a heuristic algorithm to find near-optimal solutions and provided a branch and bound algorithm to solve the problem. Okoliwski and Gawiejnowiz [11] studied the parallel machine problem that learning effect on job processing time is modeled by the general Dejong's learning curve to minimize the makespan. Hsu, Kuo and Yang [12] considered an unrelated parallel machine problem with learning effect and setup time that the setup time of each job is dependent to past sequence. They proposed a solution in polynomial time to minimize the total completion time.

In this study, unrelated parallel machine scheduling problems with the effects of aging and learning and multi maintenance activities is studied. The objective is to 
minimize the makespan. The remaining of paper is organized as follows: in section 2 the problem is described and formulated. Two polynomial algorithms are presented to solve the problem under study in section 3 . In section 4 the numerical examples are solved and finally calculations are given in section 5 .

\section{Problem Definition}

In this section, the notation that is used throughout the paper will be explained first, followed by the formulation of the problem.

$\mathrm{n}$ The total number of jobs;

$\mathrm{m}$ The total number of machines;

$t_{h}$ The duration of each maintenance activity, $\mathrm{h}=1,2, . ., \mathrm{m}$;

$C_{i}$ The completion time of job $\mathrm{j}$;

It is considered that there are $\mathrm{n}$ jobs $\mathrm{j}=\left\{j_{1}, j_{2}, . ., j_{n}\right\}$ to be processed on $m$ unrelated parallel machines $\left(M_{h}, h=1,2, . ., m\right)$.All the jobs are simultaneously available at time zero and the job preemption is not allowed. The machines can process at most one job at a time. To counteract the aging effect, the machines may require maintenance activities to improve its production efficiency, during the maintenance, the machine is stopped.

We formulated the problem under study as assigned a maintenance activity in the first position on each machine. We assumed that the first maintenance activity duration is zero. All the jobs are simultaneously available at time zero and job preemption is not allowed.

Since the objective is to minimize the makespan, we have to find simultaneously the optimal maintenance locations, the optimal maintenance frequencies and the optimal job schedule, such that the makespan of all jobs are minimized. Using the conventional notation, our problem is denoted as $R_{m} / \mathrm{ma} / C_{\max }$ where $C_{i}$ and ma in the second field represent the completion time of job $\mathrm{j}$ and the maintenance activity respectively.

We define the binary variable $(j=1,2, . ., n$ $r=1,2, . ., g \quad l=1,2, . ., p \quad g=1,2, . ., u \quad h=1,2, . ., m)$ such that $x_{j r \lg h}=1$ if job $j_{i}$ is scheduled in rth position after the gth maintenance activity on 1th reset position on machine $\mathrm{h}$ and $X_{j r \lg h}=0$ otherwise. And also $y_{g h}$ is a binary variable and $y_{g h}=1$ if gth maintenance activity scheduled on machine $M_{h}$ and $y_{g h}=0$ otherwise.

The problem can be formulated as follows:

$$
\begin{gathered}
\operatorname{Min} C_{\max } \\
\sum_{h} \sum_{g} \sum_{r} \sum_{l} x_{j r \lg h}=1 \quad \forall \mathrm{j}
\end{gathered}
$$

$$
\begin{aligned}
& y_{g h} \geq y_{g+1, h} \quad \forall \mathrm{g}>1, \mathrm{~h} \\
& \sum_{r} \sum_{l} \sum_{j} x_{j r \lg h} \leq y_{g h} \cdot M \\
& C_{j} \geq(g-1) \cdot t_{h} \\
& +\left[\sum_{j^{\prime \prime}=1}^{j^{\prime}} \sum_{r^{\prime \prime}=1}^{r^{\prime}} \sum_{l^{\prime \prime}=1}^{l^{\prime}} x_{j^{\prime \prime} r^{\prime \prime} l^{\prime \prime} g h} \cdot P_{j^{\prime \prime} h}+\alpha_{j^{\prime \prime} h}\left(r^{\prime \prime}-1\right)-b_{j^{\prime \prime} h} \cdot\left(l^{\prime \prime}-1\right)\right] \\
& -\left(1-x_{j r \lg h}\right) \cdot M \quad \forall \quad \mathrm{j}, \mathrm{r}, \mathrm{l}, \mathrm{g}, \mathrm{h} \\
& C_{\text {max }} \geq C_{j} \quad \forall \mathrm{j} \\
& \sum_{l} \sum_{j} x_{j 1 \lg h}=y_{g h} \quad \forall \mathrm{g}, \mathrm{h} \\
& \sum_{l} \sum_{j} x_{j r \lg h} \leq 1 \quad \forall \mathrm{r}, \mathrm{g}, \mathrm{h} \\
& \sum_{r} \sum_{g} \sum_{j} x_{j r \lg h} \leq 1 \quad \forall 1, \mathrm{~h} \\
& \sum_{j^{\prime}} \sum_{r^{\prime}} \sum_{l^{\prime}} \sum_{g^{\prime}} x_{j^{\prime} r^{\prime} l^{\prime} g^{\prime} h}+\sum_{j^{\prime \prime}} \sum_{r^{\prime \prime}} \sum_{l^{\prime \prime}} x_{j^{\prime \prime} r^{\prime \prime} l^{\prime \prime} g h} \geq(l-1) . \\
& x_{j r \lg h}-\left(1-x_{j r \lg h}\right) \cdot M \\
& \sum_{l} \sum_{j} x_{j r-1 \lg h} \geq \sum_{l} \sum_{j} x_{j r \lg h} \quad \forall \mathrm{g}, \mathrm{h}, \mathrm{r} \geq 1 \\
& \sum_{j} \sum_{r} \sum_{g} x_{j r l-1 g h} \geq \sum_{j} \sum_{r} \sum_{g} x_{j r \lg h} \quad \forall \mathrm{l}>1, \mathrm{~h} \\
& x_{j r \lg h} \text { and } y_{g h} \text { are binary }
\end{aligned}
$$

Constraint (1) is the objective function .Constraint (2) ensures that each job must be assigned to some position $(\mathrm{r}, \mathrm{g}, \mathrm{l}, \mathrm{h})$. Constraint (3) ensures that the assigned maintenance must be precedes all unassigned maintenance on each machine. Constraint(4) ensure that no job would be done before performing maintenance on machine $h$. constraint (5) determine the completion time of each job. Constraint(6) determines the maximum completion time that must be minimized. Constraint $(7,8)$ assumed that the maintenance activity should be happen in the first position. Constraint (8) ensures that either no job or one job is scheduled in any position $(\mathrm{r}, \mathrm{g}, \mathrm{h})$ in which $\mathrm{r} \geq 2$. Constraint $(10,11$ and 12) ensures that on each machine the assigned jobs must be precedes all unassigned jobs. Constraint (13) shows the binary variable decision. 


\section{Heuristic Algorithms}

The problem under study is NP-hard even without consideration of the learning effect, aging effect and multi-maintenance activities. [15].Thus developing efficient heuristic algorithms would be a good approach, although exact algorithms provide optimal solutions, but their long running time make them impractical in most of real-life applications, therefore genetic algorithm and the imperialist competitive algorithm are provided, before developing algorithms, we first utilize a lower bound adjusted form Pinedo[2] for assessing the performance of the heuristic.

Property 1. (Pinedo)[18] .let $\mathrm{A}=\min _{1 \leq k \leq m} \pi_{l=0}^{n} a_{l, k}$, then

$$
\mathrm{LB}=\mathrm{A}
$$

$\times \max \left\{p_{1} / s_{1},\left(p_{1}+p_{2}\right) /\left(s_{1}+s_{2}\right), \ldots \sum_{j=1}^{m-1} p_{j} / \sum_{i=1}^{m-1} s_{i}\right.$,

$\left.\sum_{j=1}^{n} p_{j} / \sum_{i=1}^{m} s_{i}\right\}$

Is a lower bound of the makespan.

\subsection{Genetic Algorithm (GA)}

For solving a parallel machine scheduling problem with the effect of aging and learning and multi maintenance activities, a genetic algorithm is proposed. The main procedures of GA for implementation are as outlined below.

\subsubsection{Chromosome representation}

For a problem of $\mathrm{n}$ jobs and $\mathrm{m}$ machines, each chromosome has $\mathrm{n}$ gens. We randomly generate the $(n . m)$ uniform numbers between $(0,1)$. The ordering of the gens represented the sequencing of jobs. For example a chromosome of $(0.4,0.3,0.9,0.1)$ in a problem with 1 machine would represent as in (fig1):

\begin{tabular}{|l|l|l|l|}
\hline$j_{3}$ & $j_{1}$ & $j_{2}$ & $j_{4}$ \\
\hline
\end{tabular}

Figure 1. job sequencing

The second binary part, decide to assign maintenance activity to gens or not. (Fig 2) illustrated an example of chromosome coding. In this fig four jobs must be performed on 2 machines, each row is assigned to one machine, as it shown $j_{2}, j_{3}$ are assigned to machine 1 and the rest to machine 2. Finally we have :

Machine 1: $j_{3} \mathrm{ma}, j_{2}$

Machine 2: $j_{1}, j_{4}$

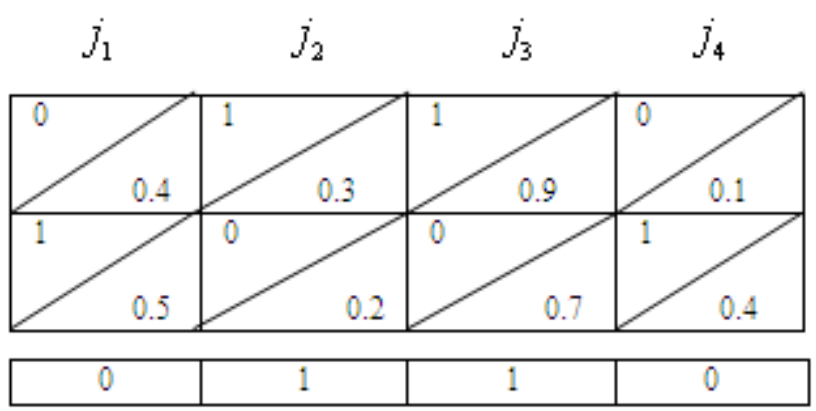

Figure 2. chromosome representation

\subsubsection{Initialization}

Generating a set of initial solution is the first step for implementing GA. The major decision in GA accomplishment is to determine the appropriate population size. If the selected number is too small, we may not be able to achieve a good solution, contrarily the larger number takes too much computational time to achieve a better solution [1]. In this proposed algorithm, the quintuple of first population size is used.

\subsubsection{Evaluation}

We calculate the fitness value of each chromosome by their objective value.

\subsubsection{Crossover}

Crossover is the method of merging the genetic information of two individuals. In this study, two methods for crossover operator are used. First is N Point crossover operator which is exchanging of $\mathrm{N}$ genes between the chromosomes of two parents.( Fig.3 A,B) illustrated the assumed parent, (Fig4) showed five random numbers are generated between $(0,1)$. If generated numbers are less than 0.5 , information of first parent will be used otherwise information of second parent would be assigned (Fig 5). The second method that applied is one point crossover operator that generated $\mathrm{m}$ uniform numbers between one and $\mathrm{n}$ to choose the cutting point (Fig.6 ).
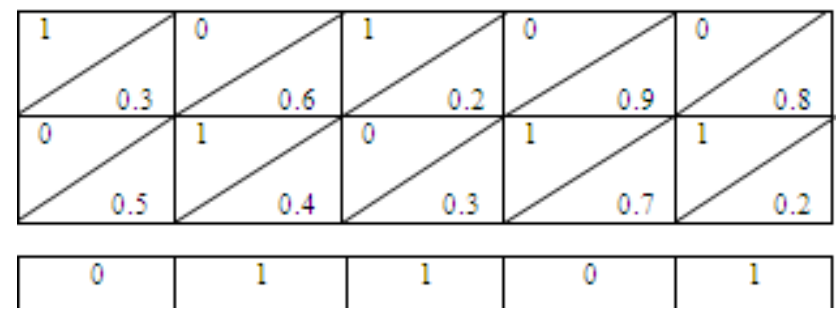

Figure 3. Assumed parent chromosome (A)
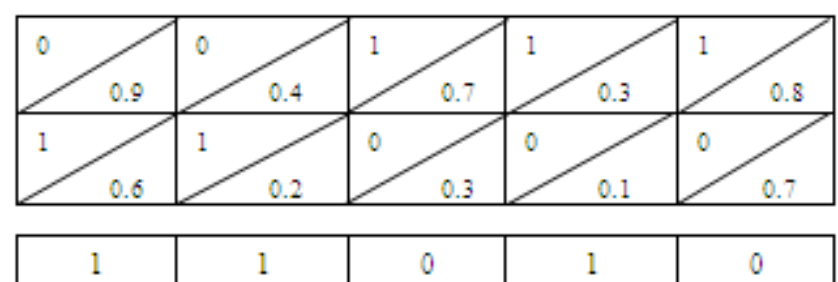

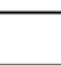

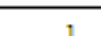

1

0

1

0

Figure 3. Assumed parent chromosome (B) 


\begin{tabular}{|l|l|l|l|l|}
\hline 0.6 & 0.1 & 0.3 & 0.8 & 0.2 \\
\hline
\end{tabular}

Figure 4. Random string
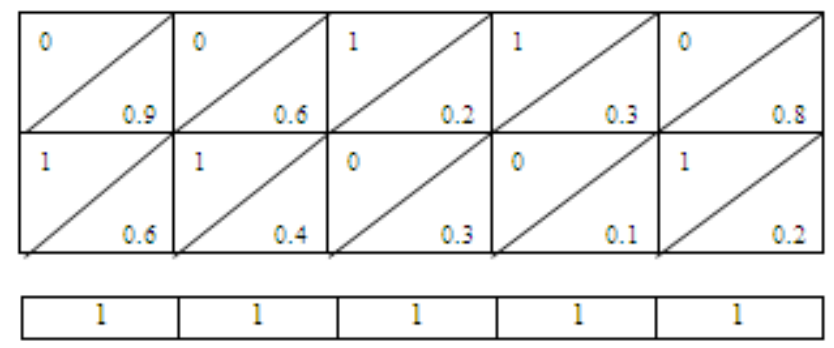

Figure 5. N point crossover
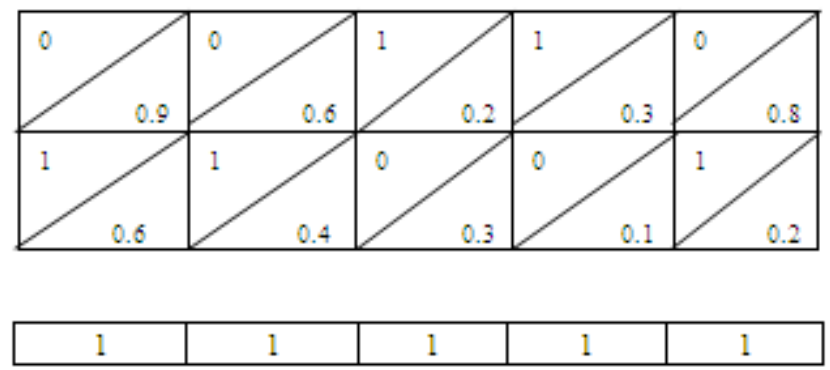

Figure 6. Cutting point crossover (cutting point=3)

\subsubsection{Mutation}

The mutation operator randomly selects individual gens and swaps its value. As shown in (Fig 7), two obtained numbers are and so offspring is generated by replacing these two gens.
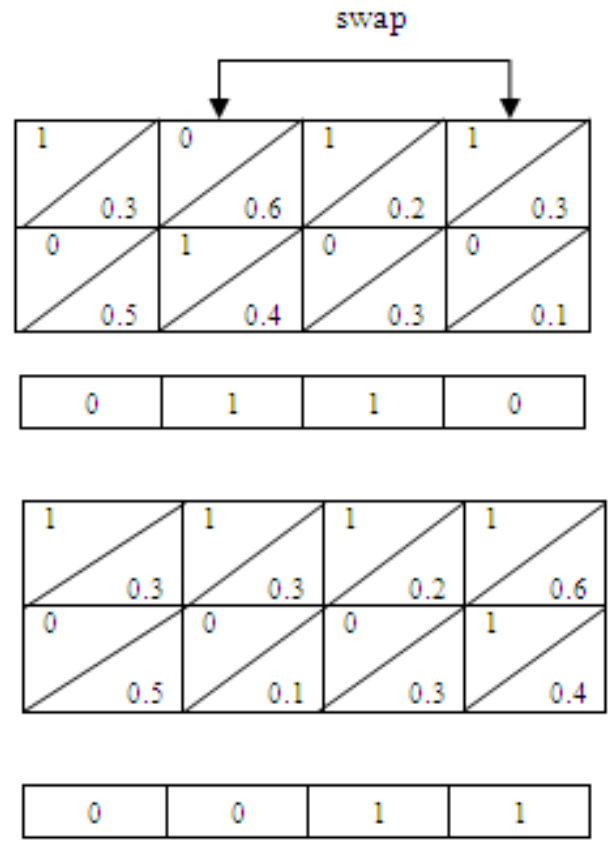

Figure 7. Mutation method

\subsection{Imperialist Competitive Algorithm}

ICA is on the most powerful evolutionary algorithms in the meta heuristic field and the computer simulation based on socio -political evolution that is developed by Atashpaz-Gargari[21]. Imperialist competitive algorithm chart is presented in Fig8. Similar to the other evolution algorithms, the ICA is started with initial random population. Each member of the population is called countries. Some of the best countries based on their cost function are categorized as the imperialist and all other countries form the colonies of the imperialist.

After all empires were formed, the competition between countries begins. First, the colonies in each of the empires start moving toward their relevant imperialist state and change the place in new position. [22]

Each empire that couldn't be success in competition would be eliminated and is considered as a colony in competition to be assimilated by other imperialist. Remaining of one emperor is the stopping point of the algorithm.

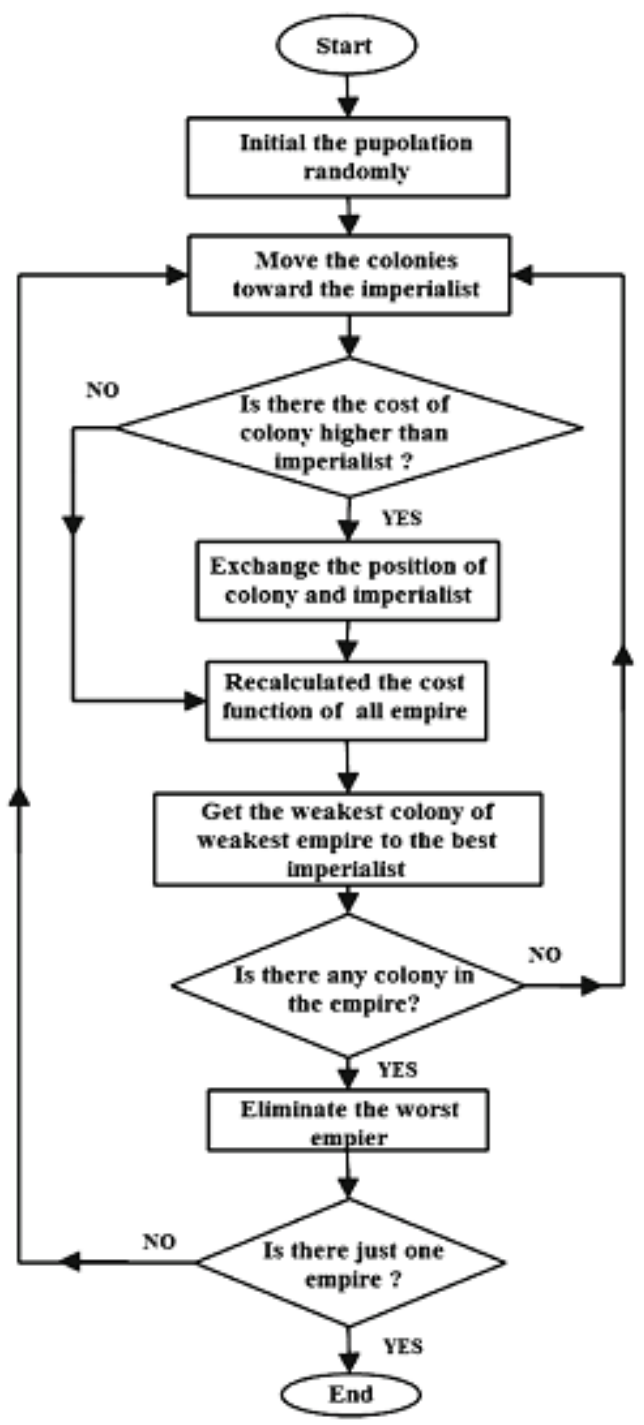

Figure 8. Imperialist competitive algorithm (ICA) flowchart[22] 


\subsection{Computational Experiments}

In this section, we conducted the results of the computational experiments to evaluate the performance of the proposed GA and ICA. We tested only one of the parameters in each level. Each test was repeated five times. The values were considered based on table 1 and 2 for the four parameters used by proposed GA and ICA respectively.

Table 1. Different level of parameters for GA

\begin{tabular}{|c|c|c|c|}
\hline Parameter & Level1 & Level2 & Level3 \\
\hline Iteration number & 100 & 150 & 200 \\
\hline Population size & 150 & 200 & 250 \\
\hline Crossover rate & 0.9 & 0.85 & 0.8 \\
\hline Mutation rate & 0.09 & 0.12 & 0.2 \\
\hline
\end{tabular}

Table 2. Different level of parameters for ICA

\begin{tabular}{|c|c|c|c|}
\hline Parameter & Level1 & Level2 & Level3 \\
\hline $\begin{array}{c}\text { Number of } \\
\text { countries }\end{array}$ & 150 & 200 & 250 \\
\hline $\begin{array}{c}\text { Number of } \\
\text { empire }\end{array}$ & 4 & 5 & 6 \\
\hline Iteration number & 300 & 400 & 500 \\
\hline $\begin{array}{c}\text { Revolution } \\
\text { possibility }\end{array}$ & 0.1 & 0.15 & 0.2 \\
\hline
\end{tabular}

\subsection{Taguchy Method}

The Taguchi method is a statistical method to achieve robust parameter design [19]. We utilize the Taguchi method to purpose the values of parameters, so table 3 and 4 shows the best value that conducted based on Taguchi method. All the algorithms are coded using MATLAB 7.5 and run on Intel Core $2.50 \mathrm{GHz} \mathrm{CPU}$ and $4 \mathrm{~GB}$ RAM on windows 7.

Table 3. GA parameters settings

\begin{tabular}{|c|c|}
\hline GA Parameters & Level \\
\hline Iteration number & 200 \\
\hline Population size & 150 \\
\hline Crossover rate & 0.9 \\
\hline Mutation rate & 0.2 \\
\hline
\end{tabular}

Table 4. ICA parameters settings

\begin{tabular}{|c|c|}
\hline ICA Parameters & Level \\
\hline Number of countries & 200 \\
\hline Number of empires & 150 \\
\hline Iteration number & 0.9 \\
\hline Revolution possibility & 0.2 \\
\hline
\end{tabular}

Table 5 represented the comparison of mathematical model and two proposed algorithm.

\section{RPD Analyzing for Proposed Heuristic Algorithms}

As it showed in (fig 9) RPDs are zero in small measures and it is an approval of accuracy for three proposed algorithms. Mathematical model is not capable of finding optimal solution in bigger measures. Also it is concluded that there is more gradient in genetic algorithm than ICA algorithm that conducted to ICA could have better efficiency than genetic algorithm.

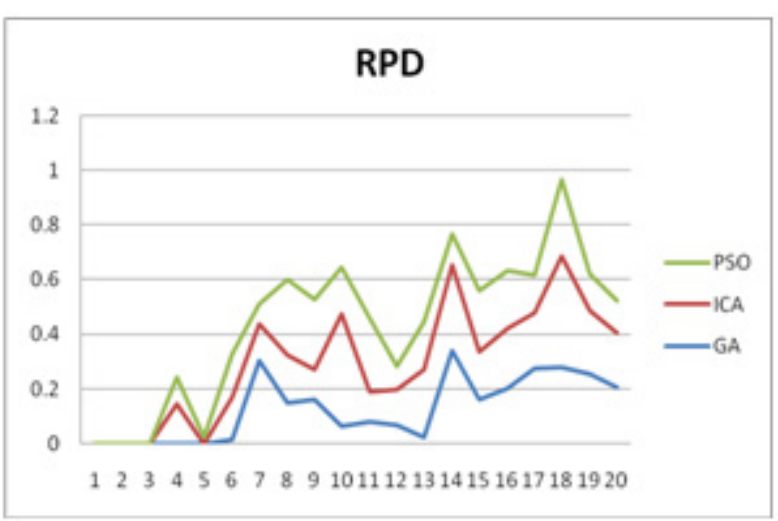

Figure 9. RPD graph for two proposed algorithm

\section{Time Analyzing for Proposed Heuristic Algorithm}

Solving time of two heuristic is studied in this section. As illustrated in (fig 10) genetic algorithm solving time has a considerable differences to ICA algorithm which is means ICA could find optimal solution in less time.

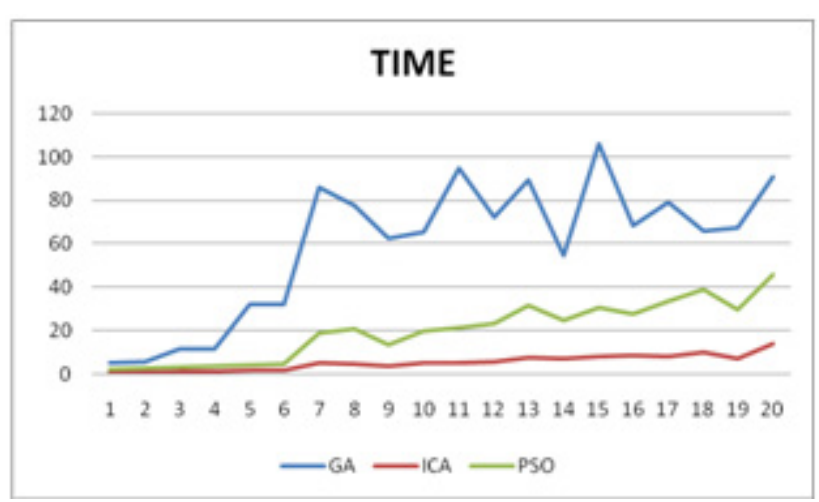

Figure 10. Time analyzing for proposed heuristic algorithm 
Table 5. Comparison of results of the model solved by lingo, the proposed GA and ICA

\begin{tabular}{|c|c|c|c|c|c|c|c|c|c|c|c|c|c|c|c|c|}
\hline \multicolumn{5}{|c|}{ mathematical model } & \multicolumn{4}{|c|}{ Genetic algorithm } & \multicolumn{4}{|c|}{ ICA algorithm } & \multicolumn{4}{|c|}{ PSO algorithm } \\
\hline no & job & machine & optimal & time & AVG & Best & Time & RPD & AVG & Best & Time & RPD & AVG & Best & Time & RPD \\
\hline 1 & 5 & 2 & 32.934 & $00: 25: 49$ & 32.934 & 32.934 & 00:00:5.7 & 0 & 32.934 & 32.934 & 00:00:0.83 & 0 & 32.934 & 32.934 & 00:00:2.32 & 0 \\
\hline 2 & 5 & 3 & 21.978 & $0: 53: 12$ & 21.978 & 21.978 & 00:00:5.59 & 0 & 21.978 & 21.978 & 00:00:0.68 & 0 & 21.978 & 21.978 & 00:00:2.66 & 0 \\
\hline 3 & 10 & 2 & - & - & 58.78 & 58.78 & 00:00:11.56 & 0 & 58.78 & 58.78 & $00: 00: 1.3$ & 0 & 58.78 & 58.78 & 00:00:3.29 & 0 \\
\hline 4 & 10 & 3 & - & - & 40.868 & 40.868 & 00:00:11.65 & 0 & 42.068 & 40.868 & 00:00:1.36 & 0.146 & 41.668 & 40.868 & 00:00:3.62 & 0.097 \\
\hline 5 & 15 & 2 & - & - & 86.384 & 86.384 & 00:00:31.9 & 0 & 87.384 & 87.384 & 00:00:1.856 & 0 & 86.784 & 86.384 & 00:00:4.42 & 0.023 \\
\hline 6 & 15 & 3 & - & - & 56.98 & 56.78 & 00:00:32.11 & 0.017 & 60.58 & 58.78 & 00:00:1.58 & 0.153 & 58.58 & 56.78 & 00:00:6.69 & 0.158 \\
\hline 7 & 30 & 5 & - & - & 72.11 & 67.97 & 00:01:42 & 0.304 & 78.03 & 75.97 & 00:00:4.94 & 0.135 & 74.337 & 73.27 & 00:00:18.91 & 0.072 \\
\hline 8 & 30 & 7 & - & - & 55.397 & 53.78 & 00:01:2.159 & 0.15 & 59.615 & 57.78 & 00:00:3.78 & 0.176 & 57.62 & 54.78 & 00:00:13.78 & 0.273 \\
\hline 9 & 35 & 5 & - & - & 84.187 & 81.538 & 00:01:17.7 & 0.162 & 91.527 & 89.538 & 00:00:4.71 & 0.111 & 91.005 & 86.584 & 00:00:20.85 & 0.255 \\
\hline 10 & 35 & 7 & - & - & 64.478 & 63.67 & 00:01:5.43 & 0.063 & 67.956 & 62.78 & 00:00:4.86 & 0.412 & 67.02 & 64.78 & 00:00:19.99 & 0.172 \\
\hline 11 & 40 & 5 & - & - & 94.708 & 93.208 & 00:01:41.2 & 0.08 & 103.433 & 101.208 & 00:00:5.17 & 0.109 & 108.74 & 95.584 & $00: 00: 21.18$ & 0.27 \\
\hline 12 & 40 & 7 & - & - & 71.952 & 70.97 & $00: 01: 21.37$ & 0.069 & 78.23 & 76.27 & 00:00:5.36 & 0.128 & 78.05 & 76.67 & 00:00:23.21 & 0.089 \\
\hline 13 & 50 & 7 & - & - & 89.125 & 88.284 & 00:02:12.47 & 0.024 & 95.025 & 90.538 & 00:00:7.6 & 0.247 & 95.976 & 92.738 & $00: 00: 31.54$ & 0.174 \\
\hline 14 & 50 & 12 & - & - & 54.457 & 53.78 & 00:01:28.19 & 0.341 & 63.54 & 59.78 & $00: 00: 7.264$ & 0.314 & 62.777 & 61.368 & $00: 00: 24.63$ & 0.114 \\
\hline 15 & 60 & 7 & - & - & 105.913 & 104.584 & 00:02:5.32 & 0.063 & 114.008 & 110.208 & 00:00:8.18 & 0.172 & 115.87 & 113.708 & $00: 00: 30.54$ & 0.227 \\
\hline 16 & 60 & 12 & - & - & 68.316 & 65.67 & 00:01:19.45 & 0.201 & 72.77 & 69.67 & 00:00:8.63 & 0.222 & 75.638 & 72.58 & 00:00:27.68 & 0.21 \\
\hline 17 & 70 & 12 & - & - & 78.784 & 74.67 & 00:01:40.86 & 0.275 & 82.897 & 79.67 & 00:00:7.89 & 0.202 & 86.643 & 84.27 & 00:00:33.81 & 0.14 \\
\hline 18 & 70 & 15 & - & - & 65.932 & 62.58 & 00:01:35.89 & 0.28 & 71.092 & 66.98 & 00:00:10.03 & 0.406 & 71.796 & 67.98 & 00:00:39.11 & 0.28 \\
\hline 19 & 100 & 12 & - & - & 67.33 & 64.08 & 00:01:38.14 & 0.254 & 74.314 & 70.97 & 00:00:7.21 & 0.235 & 74.274 & 72.38 & 00:00:29.86 & 0.13 \\
\hline 20 & 100 & 15 & - & - & 90.98 & 89.038 & $00: 02: 24.42$ & 0.109 & 96.241 & 94.384 & 00:00:13.87 & 0.198 & 99.756 & 97.438 & $00: 00: 45.83$ & 0.118 \\
\hline
\end{tabular}




\section{Conclusions}

In this paper we investigated unrelated parallel machine scheduling problems simultaneously with the effects of learning and aging and multi-maintenance activities. We aimed to find the optimal maintenance locations, the optimal maintenance frequencies and the optimal job schedule to minimize the makespan. We propose polynomial time algorithm to solve the problem. Three heuristic algorithms were proposed and the result proved the better efficiency of ICA algorithm. Further research may investigate the problem with deteriorating multi-maintenance activities and optimizing other performance measures.

\section{REFERENCES}

[1] T.Back, D.Fogel Z.Michalawecz..Handbook of evolutionary computation. Oxford: Oxford University Press. 1997.

[2] M.Pinedo. scheduling: Theory, algorithms, and systems $\left(3^{\text {rd }}\right.$ ed.). Upper Saddle River, NJ: Prentice Hall,2008.

[3] W-H.Kuo, D-L.Yang. Minimizing the makespan in a single machine scheduling problem with the cyclic process of an aging effect, Journal of the Operational Research Society 2008,59,416-420

[4] CL.Zhao, HY.Tang. Single machine scheduling with general job-dependent aging effect and maintenance activities to minimize the makespan. Applied Mathematical Modeling $2010,34,837-841$.

[5] SJ.Yang, DL.Yang. Single-machine scheduling problems with aging/deterioration effect under an optional maintenance activity consideration. INFOR2010,48,171-179.

[6] P.J.Lai, W.C.Lee. single-machine scheduling with general sum-of-processing-time-based and position-based learning effect. Omega, The International Journal of Management Science, 2011,39(5),467-471.

[7] X.G.Zhang, G.L.Yan. Machine scheduling problems with a general learning effect. Mathematical and Computer Modeling,2010, 51(1-2), 84-90.

[8] D.Wang, M.Z. Wang, J.B. Wang. Single-machine scheduling with learning effect and resource dependent processing times, Computers and Industrial Engineering,2010,59(3),458-462.

[9] T.C.E. Cheng, W.C. Lee, C.C. Wu Scheduling problems with deteriorating jobs and learning effects, Computers and Industrial Engineering, 2010,58(2),236-331.

[10] M.D.Toksary, E.Guner. Parallel machine earliness/tardiness scheduling problem under the effects of position based learning and linear/nonlinear deterioration. Computers and Operation Research, 2009,36(8),2394-2417.

[11] D.Okolowski, S.Gawiejnowicz. Exact and heuristic algorithms for parallel machine scheduling with DeJong's learning effect. Computers and Industrial Engineering,2010, 59(2), 272-279.

[12] C.J.Hsu, W.H. Kuo, D.L. Yang. Unrelated parallel machine scheduling with past-sequence-dependent setup time and learning effects. Applied Mathematical Modeling,2011, 35(3), 1492-1496.

[13] G.Mosheiov. V-shaped policies for scheduling deteriorating jobs. Operation Research,1991,39,979-991.

[14] J. N. D. Gupta, S.K.Gupta. Single facility scheduling with nonlinear processing times. Computers and Industrial Engineering, 1988,14, 387-393.

[15] J.K.lenstra, A.H.G.Rinnooy Kan, P.Brucker. Complexity of machine scheduling problems. Annals of Discrete Mathematics, 1977,1, 343-362

[16] W-C ,Lee. A note on deteriorating jobs and learning in single-machine scheduling problems. International Journal of Business and Economic 2004,3,83-89.

[17] G.Schmidt. scheduling with limited machine availability.European Journal of Operational Research 2000,121,1-15

[18] Ma Y,Chu, C,Zuo C. A survey of scheduling with deterministic machine availability constraints. Computers \& Industrial Engineering.2010,58,199-211I

[19] A.R.Yildiz. A new design optimization framework based on immune algorithm and Taguchi's method. Computer in Industry.2009, 60(8), 613-620.

[20] W-J.Chen. Minimizing number of tardy jobs on a single machine subject to periodic maintenance. Omega, 2009,37,597-599.

[21] E.Atashpaz-Gargari, C.Lucas. Imperialist Competitive algorithm: an algorithm for optimization by imperialist competition. Proceedings of the IEEE Congress on Evolutionary Computation, Singapore 4661-4667,2007

[22] T. Niknam, E. Taherian Fard, s. Ehrampoosh, A. Rousta, A new hybrid imperialist competitive algorithm on data clustering, 2011,36, 293-315. 